\title{
AN EXTENSION OF HLAWKA'S INEQUALITY
}

\author{
Sin-EI TAKAHASI, YASUJI TAKAHASHI AND SHUHEI WADA
}

Abstract. We consider an extension of Hlawka's inequality on a Hilbert space in an integral form and give an extension of Djokovic's inequality as an application of our extension.

Mathematics subject classification (1991): 26D15.

Key words and phrases: Hlawka's inequality; Djokovic's inequality; Bochner integralble Hilbert space-valued function.

\section{REFERENCES}

[1] Z. D. DJokovic, Generalizations of Hlawka's inequality, Glasnik Mat. -Fiz. Astromon. Ser. II. Drustvo Mat. Fiz. Hrvatske 18 (1963), 169-175.

[2] A. Honda, Y. OKAZAKI AND Y. TAKAHASHI, Generalization of the Hlawka's inequality, Bull. Kyushu Inst. Tech. Pure Appl. Math. 45 (1998), 9-15.

[3] H. HoRNICH, Eine Ungleichung für Vektorlangen, Math. Z. 48 (1942), 268-274.

[4] D. M. Smiley And M. F. Smiley, The polygonal inequalities, Amer. Math. Monthly 71 (1964), $755-760$ 